\title{
Ultrasound-guided treatment of common peroneal neuropathy caused by Baker's cyst: a clinical note - A case report -
}

\section{Hana Cho', Dong-Rim Kim¹, Je Jin Lee', Seung Young Lee', Yong Bum Park ${ }^{3}$, Hee Sung Kim ${ }^{4}$, and Hwa-Yong Shin ${ }^{1,5}$}

1 Department of Anesthesiology and Pain Medicine, Chung-Ang University Hospital, Seoul, ${ }^{2}$ 2nd Armored Brigade, Republic of Korea Army, Paju, Departments of ${ }^{3}$ Orthopedic Surgery and ${ }^{4}$ Pathology, Chung-Ang University Hospital, ${ }^{5}$ Department of Anesthesiology and Pain Medicine, Chung-Ang University College of Medicine, Seoul, Korea
Received June 5, 2019

Revised July 2, 2019

Accepted: July 3, 2019

\author{
Corresponding author \\ Hwa-Yong Shin, M.D., Ph.D. \\ Department of Anesthesiology and \\ Pain Medicine, Chung-Ang University \\ Hospital, 102 Heukseok-ro, Dongjak- \\ gu, Seoul 06973, Korea \\ Tel: 82-2-6299-3164 \\ Fax: 82-2-6299-2585 \\ E-mail: pain@cau.ac.kr
}

\begin{abstract}
Background: Baker's cysts are usually located in the posteromedial side of the knee and seldom cause neuropathy.

Case: We describe the rare case of a 57-year-old woman with a popliteal cyst who presented with limping gait and pain in her lower leg. She was electronically diagnosed with common peroneal neuropathy and transferred to our pain clinic. On ultrasound examination, about $2.0 \times 1.2 \mathrm{~cm}$ sized popliteal cyst was found to extend to the fibular head, compressing the common peroneal nerve. Therefore, ultrasound-guided aspiration of the cyst and a common peroneal nerve block were performed. Immediately after the procedure, the pain, dysesthesia, and limping gait were relieved. Although her pain and dysesthesia were relieved, she underwent the surgery because of limping gait.
\end{abstract}

Conclusions: In this case, we found the Baker's cyst, the cause of the common peroneal neuropathy, and treated it immediately by just simple ultrasound examination and aspiration.

Keywords: Osteoarthritis, knee; Peroneal neuropathies; Popliteal cyst; Ultrasonography.
A popliteal cyst (Baker's cyst) is a fluid-filled mass generated in the popliteal fossa. Baker's cysts are commonly developed in the posterior aspect of the knee, mostly within the gastrocnemio-semimembranosus bursa [1,2]. Baker's cysts are mainly caused by intra-articular pathologies such as osteoarthritis and meniscus tears [3]. Baker's cysts may present with various symptoms and complications. Common symptoms include swelling and pain, but many are asymptomatic $[1,3]$.

Complications include compartment syndrome, thrombophlebitis, and entrapment neuropathy. Without neuropathy, initial treatment is non-operative and is focused on pain relief. If this conservative approach fails, surgical excision could be considered [2].

Peroneal neuropathy is the third most common focal neuropathy [4]. The most significant symptom is weakness of ankle dorsiflexion $[4,5]$. The causes of peripheral neuropathy are direct nerve injury, infection, compression, and generalized diseases, like diabetes mellitus. Treatments of peroneal neuropathy are focused on symptomatic relief [5].

Although there have been many case reports on peroneal neuropathy, peroneal neuropathy caused by Baker's cyst has been rarely reported. Here, we present a case of com-

This is an Open Access article distributed under the terms of the Creative Commons Attribution Non-Commercial License (http://creativecommons.org/licenses/by-nc/4.0) which permits unrestricted non-commercial use, distribution, and reproduction in any medium, provided the original work is properly cited.

Copyright (C) the Korean Society of Anesthesiologists, 2020 
mon peroneal neuropathy, which developed due to compression of an extraneural popliteal cyst, as well as a review of the literature.

\section{CASE REPORT}

A 57-year-old woman visited the emergency room with left foot drop and mild pain, which had started suddenly 3 days prior. The pain severity was $3 / 10$ on the visual analog scale (VAS) score. The patient had a medical history of diabetes mellitus for a year and hypertension. She had received an operation of osteochondral autograft transfer on her left knee 10 years ago. On physical examination, she presented with a tingling sensation and numbness in her left lateral lower leg and foot dorsum, and was positive for Tinel's sign. The motor strength of ankle dorsiflexion and big toe extension were grade I, and the motor strength of hip flexion and hip abduction were grade IV. Upon a simple radiological study of the left knee, severe osteoarthritis was revealed. Findings were compatible with Kellgren-Lawrence grade III. There were no other remarkable findings. Common peroneal neuropathy was suspected; thus, ankle foot orthosis was applied and she was referred to the orthopedic department.

Seven days after symptom onset, she visited orthopedic department. Under the same diagnosis, $10 \mathrm{mg}$ of predniso- lone was prescribed for 3 days and electromyography on her lower leg was planned.

Seventeen days after symptom onset, a motor nerve conduction study and needle electromyography (EMG) were performed. The motor nerve conduction study showed no response of the left peroneal nerve contrary to the normal findings in both the tibial and right peroneal nerves. A sensory nerve conduction study of the left superficial and deep peroneal nerve also showed no response (Table 1).

In the needle EMG results, abnormal spontaneous activities (positive sharp waves, fibrillation potentials) were recorded in the left tibialis anterior, peroneus longus, extensor halluces longus, and extensor digitorum brevis muscle, which are innervated by the common peroneal nerve. Motor unit action potential analysis and recruitment showed no activity in these muscles. A needle EMG of the other muscles did not reveal any abnormalities (Table 2).

Considering the initial nerve conduction study and needle EMG findings, left common peroneal neuropathy around the knee level with severe partial axonotmesis or severe conduction block was highly suspected. For further evaluation, magnetic resonance imaging (MRI) study of her left knee was planned.

Meanwhile, the patient's pain was getting worse; therefore, $150 \mathrm{mg}$ of pregabalin, 1,300 mg of acetaminophen, and $150 \mathrm{mg}$ of tramadol were prescribed. One month after symptom

Table 1. Nerve Conduction Study Performed 17 Days after Symptom Onset

\begin{tabular}{|c|c|c|c|c|}
\hline Nerve & Segment & Distal latency (ms) & Amplitude (mV) & Conduction velocity $(\mathrm{m} / \mathrm{s})$ \\
\hline \multicolumn{5}{|c|}{ Motor nerve conduction study } \\
\hline Rt. Tibial & & 4.38 & 15.7 & 46 \\
\hline \multirow[t]{4}{*}{ Peroneal } & EDB & 4.64 & 0.8 & 50.9 \\
\hline & $\mathrm{TA}-4 \mathrm{~cm}$ below $\mathrm{FH}$ & 2.29 & 1.9 & - \\
\hline & $\mathrm{TA}-\mathrm{FH}$ & 2.97 & 1.7 & 59.1 \\
\hline & $\mathrm{TA}-6 \mathrm{~cm}$ above $\mathrm{FH}$ & 3.96 & 1.6 & 60.6 \\
\hline Lt. Tibial & & 4.32 & 14.4 & 43.2 \\
\hline \multirow[t]{5}{*}{ Peroneal } & EDB & NR & NR & NR \\
\hline & $\mathrm{TA}-4 \mathrm{~cm}$ below $\mathrm{FH}$ & NR & NR & NR \\
\hline & $\mathrm{TA}-\mathrm{FH}$ & NR & NR & NR \\
\hline & TA - $6 \mathrm{~cm}$ above $\mathrm{FH}$ & NR & NR & NR \\
\hline & & Distal latency (ms) & Peak latency (ms) & Amplitude $(\mu \mathrm{V})$ \\
\hline \multicolumn{5}{|c|}{ Sensory nerve conduction study } \\
\hline Rt. Superficial peroneal & & 1.25 & 1.98 & 17.2 \\
\hline Deep peroneal & & 2.6 & 3.49 & 7.3 \\
\hline Sural & & 1.82 & 2.66 & 24.4 \\
\hline Lt. Superficial peroneal & & NR & NR & NR \\
\hline Deep peroneal & & $\mathrm{NR}$ & NR & NR \\
\hline Sural & & 1.61 & 2.24 & 25.6 \\
\hline
\end{tabular}

EDB: extensor digitorum brevis, TA: tibialis anterior, FH: fibula head, NR: no response. 
onset, she was referred to our pain clinic. Physical examination findings were the same as her first visit at the emergency room; however, her pain got worse as the VAS score was $8 / 10$ compared to $3 / 10$ on her first visit.

Upon ultrasound examination of her left knee, about 2.0 $\times 1.2 \mathrm{~cm}$ sized popliteal cyst was found to extend to the fibular head, compressing the common peroneal nerve (Fig. 1). Thus, ultrasound-guided aspiration and common peroneal nerve block were performed (Fig. 2). The aspirated fluid was yellow, thick, and mucinous with a volume of $5 \mathrm{ml}$. Nerve block was performed with $0.1875 \%$ ropivacaine and $20 \mathrm{mg}$ triamcinolone. Immediately after nerve block, pain and paresthesia were relieved. We prescribed $50 \mathrm{mg}$ of pregabalin, 1,300 mg of acetaminophen, $150 \mathrm{mg}$ of tramadol, and 1,000 mg of naproxen for 3 weeks.

Six weeks after symptom onset, MRI of her left knee was

Table 2. Needle Electromyography Performed 17 Days after Symptom Onset

\begin{tabular}{|c|c|c|c|c|c|c|c|c|c|c|}
\hline & \multirow{2}{*}{ Muscles } & \multirow{2}{*}{$\begin{array}{c}\text { Insertional } \\
\text { activity }\end{array}$} & \multicolumn{4}{|c|}{ Spontaneous activity } & \multicolumn{3}{|c|}{ Voluntary MUAP } & \multirow{2}{*}{$\begin{array}{l}\text { Recruitment } \\
\text { pattern }\end{array}$} \\
\hline & & & PSW & Fib & CRD & Fasc & Amp & Dur & PPP & \\
\hline \multicolumn{11}{|c|}{ Lumbar paraspinal (both) } \\
\hline & L4-L5 & & 0 & 0 & 0 & 0 & & & & \\
\hline & L5-S1 & & 0 & 0 & 0 & 0 & & & & \\
\hline \multirow[t]{13}{*}{ Lt. } & Gluteus maximus & & 0 & 0 & 0 & 0 & $\mathrm{~N}$ & $\mathrm{~N}$ & $\mathrm{~N}$ & C \\
\hline & Gluteus medius & & 0 & 0 & 0 & 0 & $\mathrm{~N}$ & $\mathrm{~N}$ & $\mathrm{~N}$ & C \\
\hline & Tensor fascia latas & & 0 & 0 & 0 & 0 & $\mathrm{~N}$ & $\mathrm{~N}$ & $\mathrm{~N}$ & $\mathrm{C}$ \\
\hline & Vastus medialis & & 0 & 0 & 0 & 0 & $\mathrm{~N}$ & $\mathrm{~N}$ & $\mathrm{~N}$ & $\mathrm{C}$ \\
\hline & Biceps femoris (long) & & 0 & 0 & 0 & 0 & $\mathrm{~N}$ & $\mathrm{~N}$ & $\mathrm{~N}$ & $\mathrm{C}$ \\
\hline & Biceps femoris (short) & & 0 & 0 & 0 & 0 & $\mathrm{~N}$ & $\mathrm{~N}$ & $\mathrm{~N}$ & C \\
\hline & Tibialis anterior & Inc. & + & + & 0 & 0 & NA & NA & NA & NA \\
\hline & Peroneus longus & Inc. & + & + & 0 & 0 & NA & NA & NA & NA \\
\hline & Gastrocnemius (medial) & & 0 & 0 & 0 & 0 & $\mathrm{~N}$ & $\mathrm{~N}$ & $\mathrm{~N}$ & $\mathrm{C}$ \\
\hline & Tibialis posterior & & 0 & 0 & 0 & 0 & $\mathrm{~N}$ & $\mathrm{~N}$ & $N$ & $\mathrm{C}$ \\
\hline & Extensor hallucis longus & Inc. & + & + & 0 & 0 & NA & NA & NA & NA \\
\hline & Extensor digitorum brevis & Inc. & + & + & 0 & 0 & NA & NA & NA & NA \\
\hline & Abductor hallucis & & 0 & 0 & 0 & 0 & $\mathrm{~N}$ & $\mathrm{~N}$ & $\mathrm{~N}$ & $\mathrm{C}$ \\
\hline
\end{tabular}

MUAP: motor unit action potential, PSW: positive sharp waves, Fib: fibrillation potentials, CRD: complex repetitive discharge, Fasc: fasciculation, Amp: amplitude, Dur: duration, PPP: polyphasic potentials, o: none, +: weakly positive, Inc.: increased, N: normal, C: complete, NA: no activity.

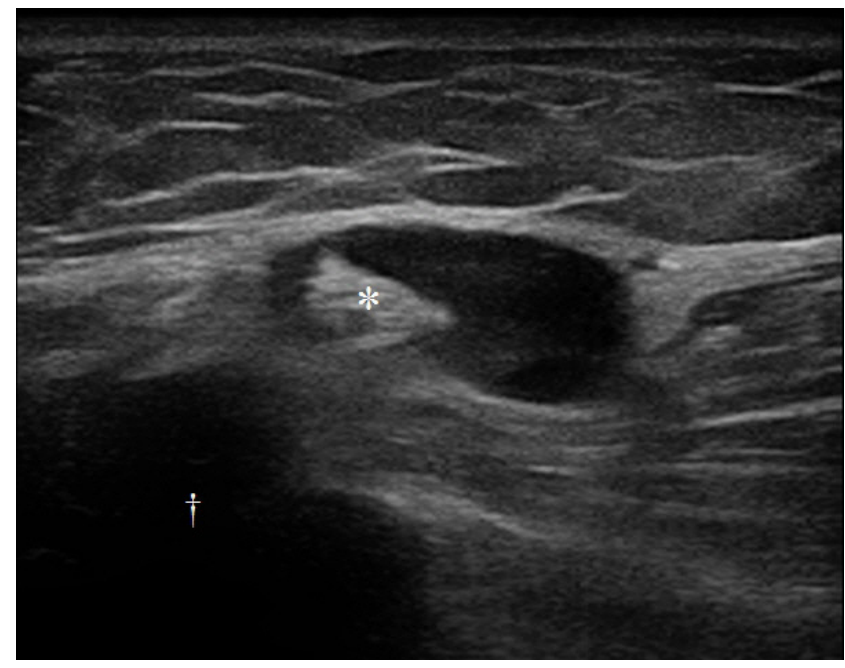

Fig. 1. Ultrasound images of the Baker's cyst. It follows the uncommon route, which arises from the popliteal space and extends to the fibular head (dagger) posterior to lateral femoral condyle compressing the common peronaeal nerve (asterisk).

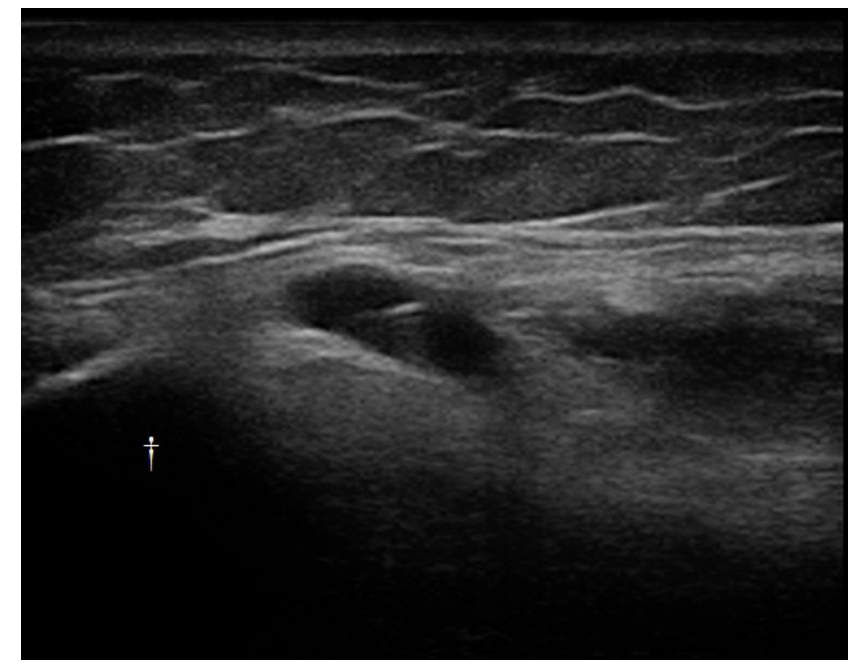

Fig. 2. An ultrasound image of the aspiration of the Baker's cyst, which arises from the popliteal space and extends to the fibular head (dagger) 


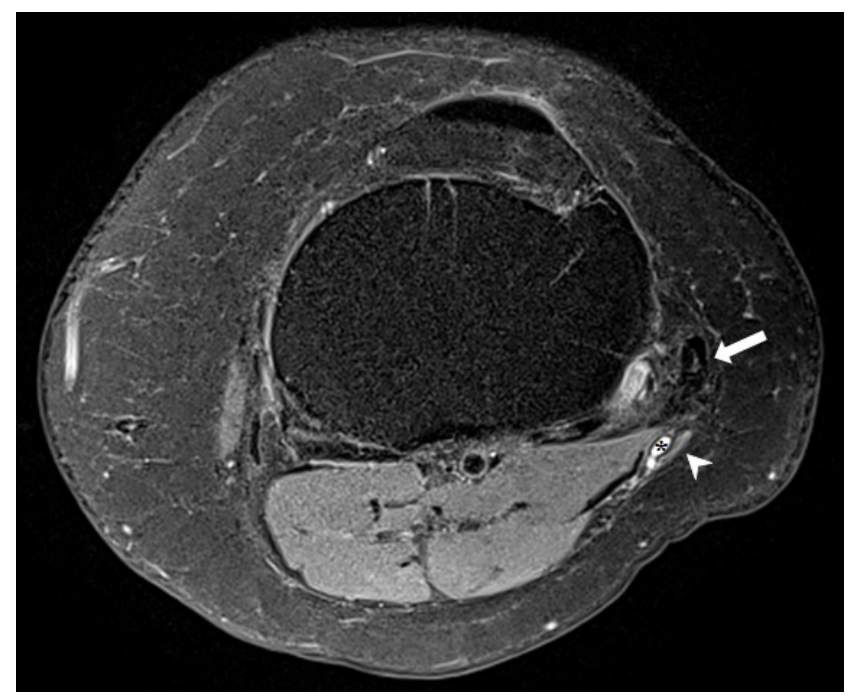

Fig. 3. Axial proton density-weighted magnetic resonance imaging with fat suppression of the knee. Cystic lesion (asterisk) compressing the common peroneal nerve (arrowhead) at the level of the fibular head (arrow).

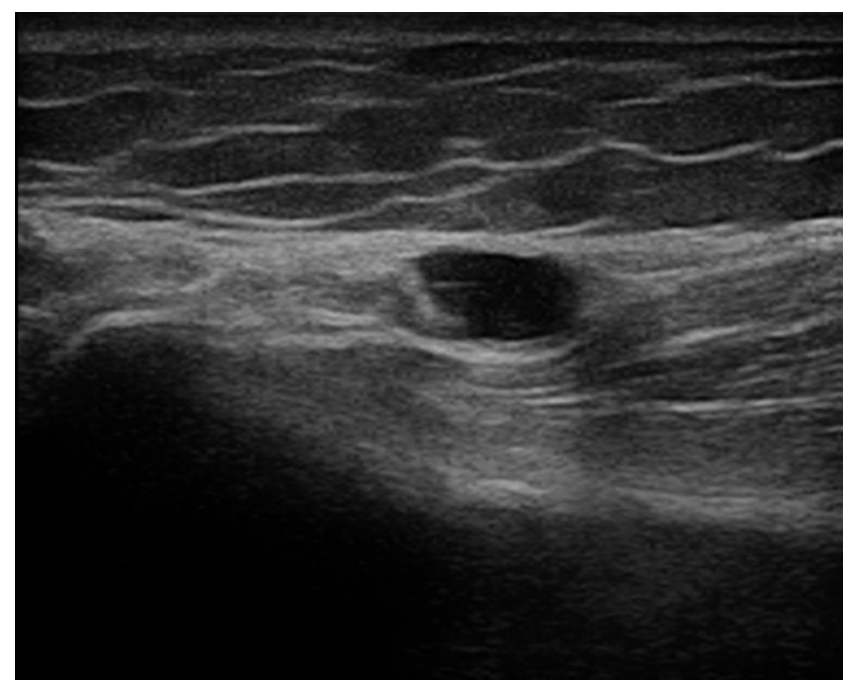

Fig. 5. An ultrasound image of the aspiration of the Baker's cyst at the second visit.

performed (Fig. 3). Axial proton density-weighted MRI with fat suppression of the knee showed that the cystic lesion was compressing the common peroneal nerve at the level of the fibular head. In addition, there was an increased signal intensity in the proximal anterior compartment of lower leg, which indicated denervation injury (Fig. 4). The menisci showed a degenerative change without tear, and bone and cartilage imaging showed findings compatible with severe osteoarthritis.

Seven weeks after symptom onset, pain severity was $0 / 10$ on VAS score, but limping gait was still maintained. There-

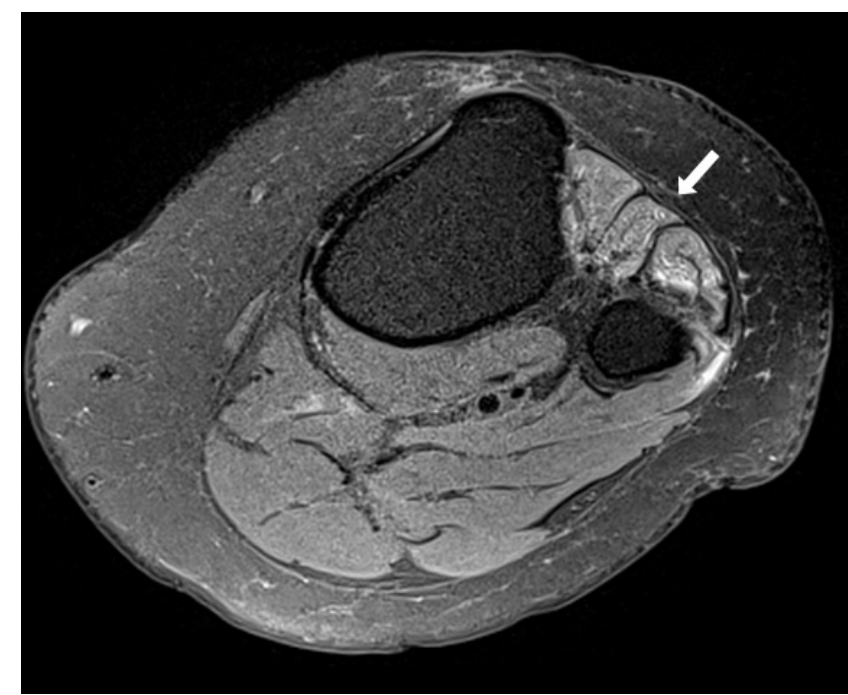

Fig. 4. Axial proton density-weighted magnetic resonance imaging with fat suppression of the knee. Increased signal intensity (arrow) in proximal anterior compartment of lower leg.

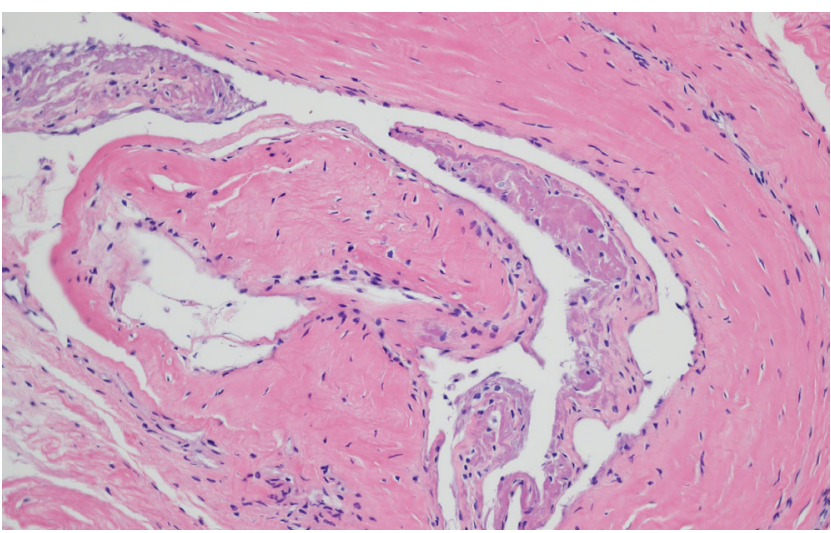

Fig. 6. Pathologic findings of the cyst excised from the knee revealed synovial lining, and the diagnosis was Baker's cyst (H\&E, $\times 200$ ).

fore, a second common peroneal nerve block were performed with $0.1875 \%$ ropivacaine and triamcinolone $20 \mathrm{mg}$ at her second visit to our pain clinic (Fig. 5). The motor strength of ankle dorsiflexion and big toe extension were grade II, even though the surgical removal of the perineural cyst was determined at the orthopedic department.

Ten weeks after symptom onset, surgical excision of the cyst was performed and pathologic findings revealed that cyst was a Baker's cyst (Fig. 6). Four months after surgery, she had no pain but the sensorimotor deficit remained.

Informed consent to publication are obtained from the patient. 


\section{DISCUSSION}

Distention of the popliteal bursa, such as the gastrocnemio-semimembranosus bursa, is known as Baker's cyst [13]. Baker's cyst is usually connected to the knee joint by a valvular opening, and is commonly associated with intra-articular diseases and inflammation, such as meniscus tears, rheumatoid arthritis, and osteoarthritis [1,2]. Even though the most common location of a popliteal cyst is between the gastrocnemius and semimembranosus, there are a few case reports of the lateral occurrence of popliteal cysts [6]. Usually, Baker's cyst presents with an asymptomatic course or mild pain; however, it can sometimes present with moderate to severe complications, including compartment syndrome, thrombophlebitis, and entrapment neuropathy [2]. Diagnosis is based on the patient's past medical history, pathological examination, and imaging workup such as MRI [2,3]. If there is no neuropathological complication, only conservative treatment is required $[2,3]$.

In our case, it was not easy to reveal the cause of neuropathy due to the uncommon location of the Baker's cyst, which was located on the lateral side of popliteal area. Because Baker's cyst is usually asymptomatic, her severe pain and muscle weakness could have made diagnosis difficult. She was initially diagnosed based on just her symptoms and EMG results without knowing the exact cause. Thus, the focus of the treatment was symptom relief. When she first came to our pain clinic, she had already had motor weakness. So we considered about surgical treatment. However, she was referred from orthopedic surgery only for pain control and was scheduled to revisit there soon. Therefore we did not consider surgical treatment in our department.

The common peroneal nerve arises from the sciatic nerve, which is formed from the L4-S4 ventral rami. This nerve originates from the upper angle of the popliteal fossa and travels along the medial border of the biceps femoris. It then turns around the head of the fibula, and it is divided into the deep peroneal nerve and superficial peroneal nerve $[4,5]$. The deep peroneal nerve innervates sensory information between the first and second toe, and has motor control of toe extension and ankle dorsiflexion. The superficial peroneal nerve provides sensory information of the foot dorsum and lateral calf, and has motor control of ankle eversion $[4,5,7]$. Peroneal neuropathy is associated with various causes, such as direct nerve damage, nerve compression, nerve traction, and metabolic syndrome
$[4,5]$. Peroneal neuropathy could present with diverse symptoms depending on where the nerve is affected, but symptoms are mostly sensory loss and foot drop $[5,7,8]$. For this reason, we could identify the location of the injury based on the clinical findings. The assessment includes electrodiagnostic examinations, which included a motor conduction study, sensory conduction study, and needle EMG. Imaging studies such as MRI is also helpful $[4,5,9]$. Treatments differ from medication to surgery according to causes and symptoms.

In the present case, our patient suffered from foot drop, paresthesia, and mild pain. On the physical examination, the motor grade was I on ankle dorsiflexion and big toe extension. Numbness and tingling sensation were observed on the common peroneal nerve territory. Also, the electrodiagnostic result was indicative of common peroneal neuropathy. To find out the cause of common peroneal neuropathy, we performed an ultrasound examination. We placed the probe on the lateral side of her left knee to examine the nerve pathway and lesion and surprisingly found a huge cyst near the left fibular head. Thus, we thought that the cause of neuropathy was compression by a cystic lesion. To relieve the compression, we aspirated the cystic fluid and blocked the common peroneal nerve; the patient experienced dramatic reliefs after the procedure. Soon, pain severity reduced to $0 / 10$ on the VAS score. What we missed was the pathological examination of the aspirated fluid. In fact, the lateral presentation of a popliteal cystic lesion should be diagnosed fast to rule out a meniscal cyst and soft tissue neoplasm [2]. If the examination had been performed, the diagnosis would have made earlier. Despite the unusual location, we assumed that the cystic lesion would be Baker's cyst for a number of reasons. First, ultrasound examination of her left knee, we find a large popliteal cyst was extend to the fibular head. This cyst was most likely to be a ganglion, but we could not exclude the Baker's cyst. Second, the characteristics of the aspirated fluid were the same as that of the synovial fluid, i.e., yellow, thick, and mucinous. Third, she had a medical history of knee surgery, severe osteoarthritis, and diabetes mellitus. The treatment was rapid, so we thought the treatment would be effective. However, the motor recovery was slower than our expectation, so we planned to do surgery. We were at least able to achieve the goal of pain relief.

Like most other diseases, eliminating the cause is the best treatment for common peroneal neuropathy. In this aspect, since ultrasound is easily and immediately applica- 
ble, diagnosable, and a treatable device, it would be the best choice to examine common peroneal neuropathy and other types of peripheral neuropathy. Furthermore, it is cost effective.

In our case, using ultrasound, we easily found the Baker's cyst at the level of the fibular head, which was causing the neuropathy. If the diagnosis was faster, perfect recovery of the nerve may have been achieved using only the ultrasound-guided procedure. It is a good idea to perform a minimal examination in consideration of the general location and condition of the lesion. We think performing ultrasound examination on peripheral neuropathy patients to examine various lesions could be a good option. Even if the ultrasound-guided procedure does not allow for perfect recovery of the nerve, it could at least provide pain relief and satisfaction to patients waiting for surgery or examination, like that in our case.

\section{CONFLICTS OF INTEREST}

No potential conflict of interest relevant to this article was reported.

\section{AUTHOR CONTRIBUTIONS}

Conceptualization: Hwa-Yong Shin. Data acquisition: Hana Cho, Yong Bum Park, Hee Sung Kim. Formal analysis: Hee Sung Kim. Funding: Hwa-Yong Shin. Supervision: Hwa-Yong Shin. Writing_original draft: Hana Cho, Seung Young Lee. Writing_review \& editing: Hana Cho, DongRim Kim, Je Jin Lee, Seung Young Lee.

\section{ORCID}

Hana Cho, https://orcid.org/0000-0002-9077-9972

Dong-Rim Kim, https://orcid.org/0000-0001-6448-783X

Je Jin Lee, https://orcid.org/0000-0002-9986-9026

Seung Young Lee, https://orcid.org/0000-0003-2734-1342

Yong Bum Park, https://orcid.org/0000-0002-3741-2311

Hee Sung Kim, https://orcid.org/0000-0002-8154-2391

Hwa-Yong Shin, https://orcid.org/0000-0002-8721-3070

\section{REFERENCES}

1. Herman AM, Marzo JM. Popliteal cysts: a current review. Orthopedics 2014; 37: e678-84.

2. Frush TJ, Noyes FR. Baker's cyst: diagnostic and surgical considerations. Sports Health 2015; 7: 359-65.

3. Demange MK. Baker's cyst. Rev Bras Ortop 2015; 46: 630-3.

4. Marciniak C. Fibular (peroneal) neuropathy: electrodiagnostic features and clinical correlates. Phys Med Rehabil Clin N Am 2013; 24: 121-37.

5. Baima J, Krivickas L. Evaluation and treatment of peroneal neuropathy. Curr Rev Musculoskelet Med 2008; 1: 147-53.

6. Manik P, Vasudeva N. Unusual lateral presentation of popliteal cyst: a case report. Nepal Med Coll J 2006; 8: 284-5.

7. Masakado Y, Kawakami M, Suzuki K, Abe L, Ota T, Kimura A. Clinical neurophysiology in the diagnosis of peroneal nerve palsy. Keio J Med 2008; 57: 84-9.

8. Ferraresi S, Garozzo D, Buffatti P. Common peroneal nerve injuries: results with one-stage nerve repair and tendon transfer. Neurosurg Rev 2003; 26: 175-9.

9. Lee DI. New paradigm for the neuropathic pain. Korean J Pain 2004; 17: 99-103. 